OPEN ACCESS

Edited by:

Markus Ritter,

Paracelsus Medical University, Austria

Reviewed by:

Yangchao Chen,

The Chinese University of Hong Kong,

China

Feiyue Xu,

The Chinese University of Hong Kong,

China

Youjia Li,

Shenzhen Bay Laboratory, China

*Correspondence:

Hongwei Zhu

zhw_0509@yeah.net

Xiao Yu

yuxiaoyx4@126.com

${ }^{+}$These authors have contributed equally to this work

Specialty section:

This article was submitted to

Cell Death and Survival,

a section of the journal

Frontiers in Cell and Developmental

Biology

Received: 22 November 2021

Accepted: 17 January 2022

Published: 10 February 2022

Citation:

Chen D, Gao W, Zang L, Zhang X, LiZ,

Zhu $H$ and Yu X (2022) Ferroptosis-

Related IncRNAs Are Prognostic

Biomarker of Overall Survival in

Pancreatic Cancer Patients.

Front. Cell Dev. Biol. 10:819724.

doi: 10.3389/fcell.2022.819724

\section{Ferroptosis-Related IncRNAs Are Prognostic Biomarker of Overall Survival in Pancreatic Cancer Patients}

\author{
Dongjie Chen ${ }^{1 \dagger}$, Wenzhe Gao ${ }^{1 \dagger}$, Longjun Zang ${ }^{1}$, Xianlin Zhang ${ }^{2}$, Zheng $\mathrm{Li}^{2}$, Hongwei Zhu ${ }^{1 *}$ \\ and Xiao $\mathrm{Yu}^{1 *}$ \\ ${ }^{1}$ Department of Hepatopancreatobiliary Surgery, The Third Xiangya Hospital, Central South University, Changsha, China, \\ ${ }^{2}$ Department of General Surgery, Affiliated Renhe Hospital of China Three Gorges University, Yichang, China
}

Pancreatic cancer (PC) is one of the most lethal malignancies, the mortality and morbidity of which have been increasing over the past decade. Ferroptosis, a newly identified irondependent cell death pattern, can be induced by iron chelators and small lipophilic antioxidants. Nonetheless, the prognostic significance of ferroptosis-related IncRNAs in PC remains to be clarified. We obtained the IncRNA expression matrix and clinicopathological information of PC patients from The Cancer Genome Atlas (TCGA) and the International Cancer Genome Consortium (ICGC) datasets in the current study. Firstly, we conducted Pearson correlation analysis to delve into the ferroptosis-related IncRNAs, and univariate Cox analysis was implemented to examine the prognostic values in PC patients. Twenty-three prognostic ferroptosis-related IncRNAs were confirmed and loaded into the least absolute shrinkage and selection operator Cox (LASSO-Cox) analysis, then a ferroptosis-related IncRNA prognostic marker (Fe-LPM) was established in the TCGA dataset. Risk scores of patients were calculated and segregated PC patients into low-risk and high-risk subgroups in each dataset. The prognostic capability of Fe-LPM was also confirmed in the ICGC dataset. Gene set enrichment analysis (GSEA) revealed that several ferroptosis-related pathways were enriched in low-risk subgroups. Furthermore, we adopted a multivariate Cox regression to establish a nomogram based on risk score, age, pathological $T$ stage and primary therapy outcome. A competing endogenous RNA (ceRNA) network was also created relied on four of the twenty-three ferroptosis-related IncRNAs. In conclusion, the eight Fe-LPM can be utilized to anticipate the overall survival (OS) of PC patients, which are meaningful to guiding clinical strategies in PC.

Keywords: pancreactic cancer, ferroptosis, long non coding RNA, prognostic biomarker, ceRNA network

\section{INTRODUCTION}

Pancreatic cancer (PC) is one kind of the most devastating carcinoma worldwide (Vincent et al., 2011). Although the therapeutic strategies of PC have been developed, widespread metastases and drug resistance remain to be challenges for clinicians. What's more, the overlapping symptoms and long latency period hampered the early diagnosis of PC. These factors result in PC having a typically poor prognosis (Wolfgang et al., 2013). Hence, it is imperative to hunt for novel clinical regimens for PC patients (Dreyer et al., 2017; Neoptolemos et al., 2018). 


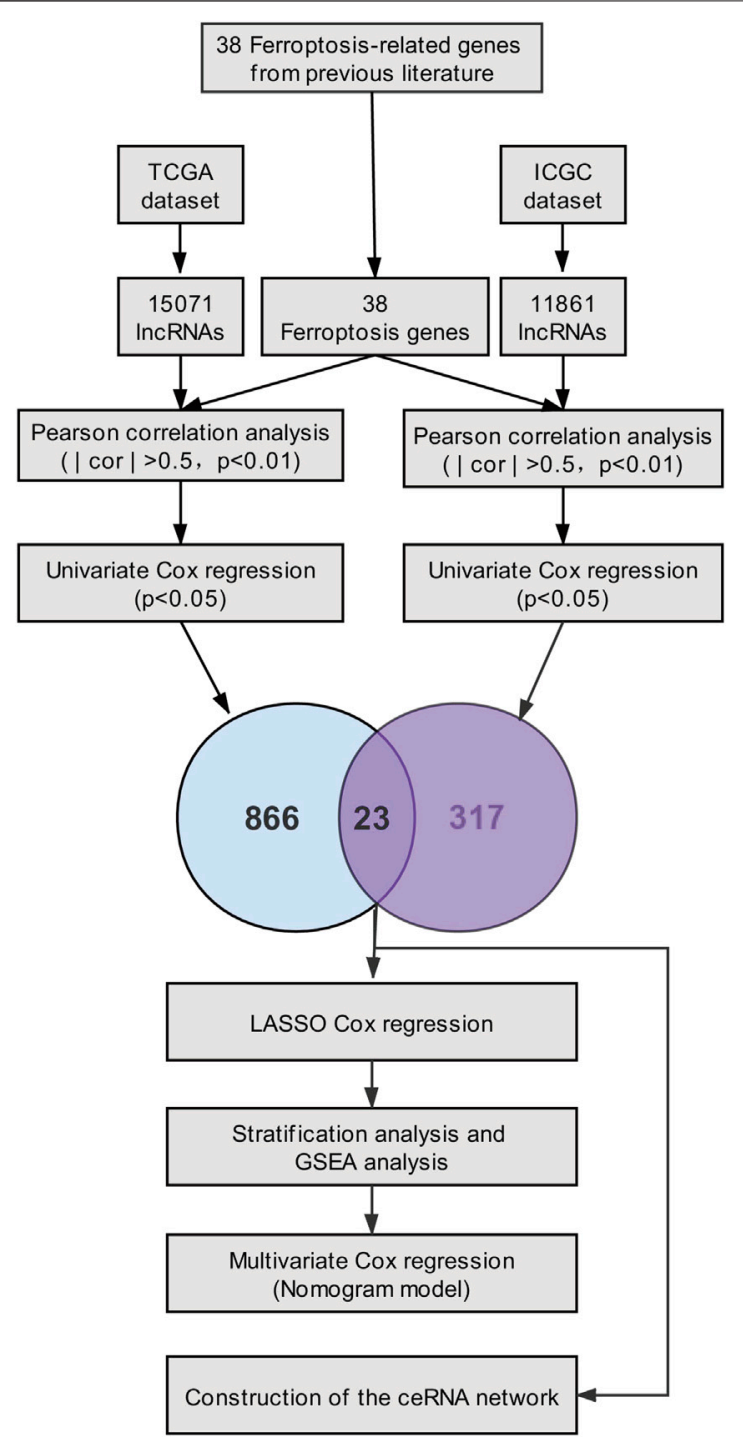

FIGURE 1 | Flow diagram of the study.

Ferroptosis, a unique type of cell death distinct from other regulated cell death (RCD), plays a pivotal role in iron metabolism and lipid peroxidation (Zheng and Conrad, 2020; Tang et al., 2021). Since the first identification of ferroptosis in 2012, numerous studies have revealed that it can regulate the pathogenesis of various human malignancies (Stockwell et al., 2020). For instance, GPX4 overexpression or ACSL4 depletion in glioblastoma cells could promote tumor necrosis via neutrophilinduced ferroptosis (Yee et al., 2020), and CAFs were reported to inhibit ferroptosis in gastric cancer cells by targeting ALOX15 and blocking lipid-ROS accumulation (Zhang et al., 2020). Lately, some researchers have elucidated that the dysfunction of ferroptosis regulators is tightly associated with the progression of PC (Dai et al., 2020).

Long non-coding RNA (lncRNA) is a class of non-coding RNA whose length exceeds 200 nucleotides. With the deep recognition of lncRNAs, it is also believed that the aberrant expression and mutation of lncRNAs serve a crucial part in the malignant proliferation of PC (Xiong et al., 2017; Sun and $\mathrm{Ma}, 2019)$. For example, PLACT1 was previously reported to be an E2F1-mediated lncRNA promoting pancreatic ductal adenocarcinoma (PDAC) tumorigenicity and metastatic potential (Ren et al., 2020); THAP9-AS1 was considered as a potential biomarker to play an essential role in PDAC growth via enhancing YAP signaling (Li et al., 2020), and overexpression of GSTM3TV2 could strengthen the chemoresistance in PC (Xiong et al., 2019). However, few studies have focused on the mechanisms of how ferroptosis regulates the development and advance in lncRNA-mediated PC. On that account, fathoming out the relationship between ferroptosis of lncRNA and PC development is urgently needed to detect novel prognostic signatures that can behave as effective targets for treatment.

In our study, based on the TCGA cohort and the ICGC cohort of PC patients, twenty-three prognostic ferroptosis-related lncRNAs were identified as prognostic ferroptosis-related lncRNAs. Then, we constructed a ferroptosis-related lncRNA prognostic marker (Fe-LPM) according to the predictive capacity of eight ferroptosis-related lncRNAs. In this way, PC patients were categorized as high-risk and low-risk subgroups, and we found many signal-transduction pathways converged on the lowrisk subgroup according to GSEA analysis. Moreover, a competing risk nomogram model was established to predict the long-term survival of patients with PC. Last but not least, we constructed a ceRNA network to search the target miRNAs and mRNAs of these ferroptosis-related prognostic lncRNAs. The flowchart of the study was shown in Figure 1.

\section{MATERIALS AND METHODS}

\section{Data Processing}

RNA-expression matrix and clinicopathological data of patients were extracted from the TCGA_PAAD (source: https://portal. gdc.cancer.gov/; $n=178$ ) as a training set, and the data in ICGC_ AU_PAAD (source: https://daco.icgc.org/; $n=91$ ) were obtained from the ICGC website as a validation set. For ensuring the comparability between TCGA and ICGC datasets and removing the batch effect, TPM (Transcripts Per kilobase of exon model per Million mapped reads) data of RNA-expression matrix were log2 transformed and normalized via "limma" R package . Patients with absent prognostic information were excluded from our analysis. Additionally, based on previous literature (Stockwell et al., 2017; Hassannia et al., 2019), we retrieved the expression matrix of 38 ferroptosis-related genes from the TCGA and ICGC datasets.

\section{Generation and Assessment of the Ferroptosis-Related IncRNA Prognostic Marker}

Aimed at identifying the ferroptosis-related prognostic lncRNAs, Pearson correlation analysis was first implemented $(\mid$ cor $\mid>0.5$, $p<0.01)$, and we performed univariate Cox analysis among TCGA and ICGC datasets separately. The ferroptosis-related 

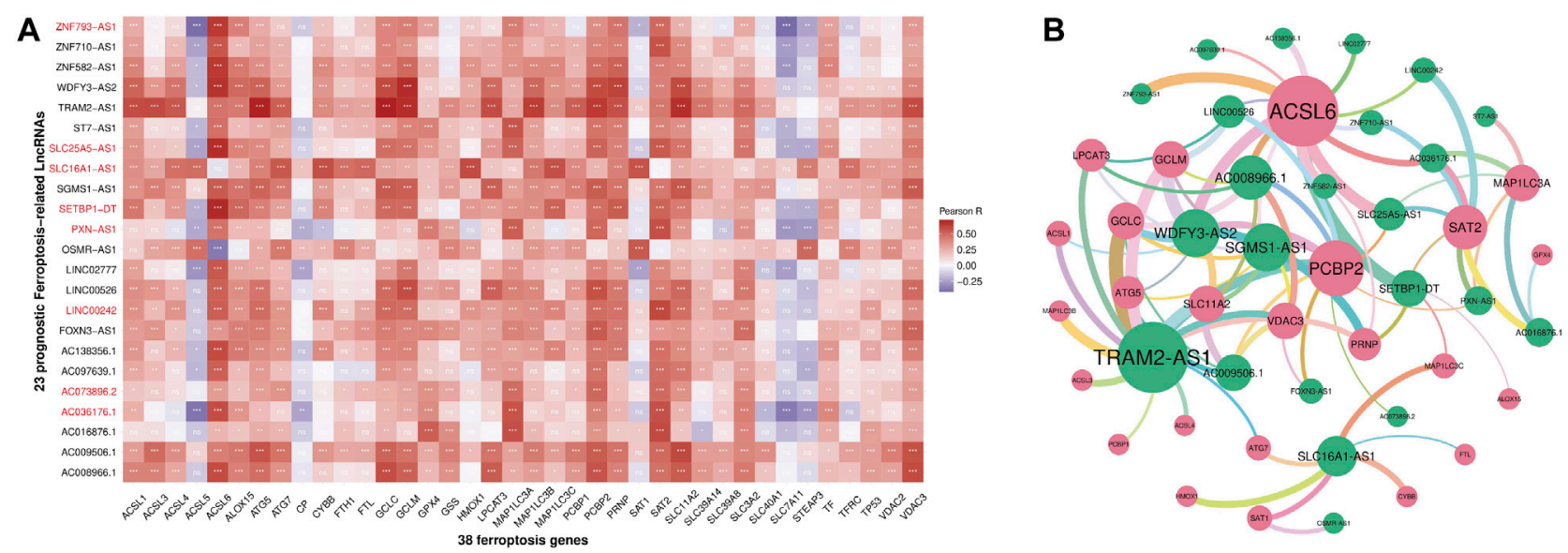

FIGURE 2 | Heatmap (A) and correlation network (B) between 38 ferroptosis-related genes and 23 prognostic ferroptosis-related IncRNAs (ns: $p \geq 0.05$, ${ }^{\star} p<0.05$, $\left.{ }^{\star \star} p<0.01,{ }^{\star \star \star} p<0.001\right)$.

lncRNAs extracted from two datasets were converged to seize the twenty-three prognostic ferroptosis-related lncRNAs. Afterward, The R package "glmnet" was used to conduct the LASSO-Cox analysis. LASSO model which performs both variable selection and regularization can shrink coefficients to zero. 10-time crossvalidation was performed to access the optimal values for $\lambda$, and the minimum $\lambda$ was selected. Then, we established a ferroptosisrelated lncRNA prognostic marker (Fe-LPM) for the PC patient implicating eight ferroptosis-related lncRNAs. The formula of the risk score was constructed as follows:

$$
\text { Risk score }=\sum_{i=1}^{n} \operatorname{Coef}_{i}{ }^{\star} x_{i}
$$

Where $\operatorname{Coe}_{i}$ represents the coefficients, $x_{i}$ represents the normalized count of each ferroptosis-related lncRNAs. We performed the Principal component analysis (PCA) to check the identifiable capability of the Fe-LPM with the "gmodels" R package. Patients were divided into high-risk and low-risk subgroups on the threshold of median risk scores. Owing to analyzing the survival conditions of Fe-LPM, the optimal cut-off value was calculated using the $\mathrm{R}$ package "survminer". The $\mathrm{R}$ package "timeROC" was applied to perform a time-dependent receiver operating characteristic (ROC) curve to estimate the forecasting capacity of the Fe-LPM. We conducted the univariate and multivariate Cox analyses to identify the independence of the Fe-LPM in predicting OS of PC patients. Based on the result of multivariate Cox regression, we developed a nomogram, and the calibration plot validated the robustness of the nomogram.

\section{Establishment of a Competing Endogenous RNA Network}

For understanding the co-expression network of lncRNAs, miRNAs and mRNAs, four of twenty-three ferroptosis-related lncRNAs were picked to predict twenty-seven target miRNAs employing miRcode database. Then, we identified fifty-seven target mRNAs of twenty-seven miRNAs based on the MiRDB, miRTarBase, StarBase and TargetScan databases. The ceRNA network was visualized with "Cytoscape" software (version 3.8.2).

\section{Pathway Enrichment Analysis and Gene Set Enrichment Analysis Analysis}

Based on the TCGA_PAAD training set, differential analysis was conducted, and then 1213 differentially expressed mRNAs (DEmRNAs) between high-risk and low-risk groups were selected according to the criteria of $\mid \log 2$ (Fold Change) $\mid>1$ and $\mathrm{p}<0.05$ utilizing the "DESeq2" R package. The 1213 DEmRNAs and the 57 target mRNAs in the ceRNA network were individually imported into the "Metascape" website for pathway enrichment analysis. Gene Set Enrichment Analysis (GSEA) was also performed to interrogate the potential pathways enriched in the high-risk and low-risk subgroups, using GSEA software (version 4.1.0). Gene expression data from the TCGA_PAAD cohort were loaded into GSEA, and the c2.cp.kegg.v7.2.symbols.gmt and h.all.v7.2.symbols.gmt were elected as the gene set database. The benchmark for which to be significantly enriched include nominal $p$-value $<0.05$, normalized enrichment score $(\mathrm{NES})>1$, and false discovery rate (FDR) $q$-value $<0.25$.

\section{Statistical Analysis}

The student's $t$-test was used to compare the distribution of risk scores. Pearson correlation test was utilized to calculate the relationships between ferroptosis-related genes and lncRNAs. Chi-square test was performed to compare the categorical data of distinct groups. Wilcoxon test was used to compare ranked and independent data between different groups. Kaplan-Meier (KM) analysis with a log-rank test was used to compare the OS between different groups. All statistical analyses were performed using the $\mathrm{R}$ programming language (Version 4.0.3). Unless expressly stated, 
TABLE 1 | The 23 ferroptosis-related prognostic IncRNAs.

\begin{tabular}{|c|c|c|c|c|c|c|c|c|}
\hline \multirow[t]{2}{*}{ IncRNA } & \multicolumn{4}{|c|}{ TCGA } & \multicolumn{4}{|c|}{ ICGC } \\
\hline & HR & HR.95L & HR.95H & $p$-value & HR & HR.95L & HR.95H & $p$-value \\
\hline OSMR-AS1 & 1.4915 & 1.0714 & 2.0763 & 0.0179 & 1.2615 & 1.0116 & 1.5732 & 0.0392 \\
\hline SLC16A1-AS1 & 1.4379 & 1.0252 & 2.0166 & 0.0354 & 1.2242 & 1.0028 & 1.4945 & 0.0469 \\
\hline SETBP1-DT & 0.8096 & 0.6639 & 0.9872 & 0.0369 & 0.7414 & 0.6194 & 0.8874 & 0.0011 \\
\hline AC097639.1 & 0.8032 & 0.6709 & 0.9617 & 0.0170 & 0.7965 & 0.6595 & 0.9621 & 0.0182 \\
\hline TRAM2-AS1 & 0.7956 & 0.6417 & 0.9863 & 0.0370 & 0.7370 & 0.5433 & 0.9998 & 0.0498 \\
\hline FOXN3-AS1 & 0.7658 & 0.6139 & 0.9552 & 0.0179 & 0.7542 & 0.5733 & 0.9921 & 0.0437 \\
\hline ZNF793-AS1 & 0.7639 & 0.6051 & 0.9645 & 0.0236 & 0.7920 & 0.6888 & 0.9107 & 0.0011 \\
\hline LINC02777 & 0.7626 & 0.5860 & 0.9923 & 0.0437 & 0.8125 & 0.6668 & 0.9900 & 0.0395 \\
\hline LINC00526 & 0.7624 & 0.5873 & 0.9898 & 0.0417 & 0.7570 & 0.6133 & 0.9345 & 0.0096 \\
\hline SLC25A5-AS1 & 0.7556 & 0.5987 & 0.9535 & 0.0182 & 0.6088 & 0.4681 & 0.7917 & 0.0002 \\
\hline ZNF582-AS1 & 0.7524 & 0.5734 & 0.9871 & 0.0400 & 0.8326 & 0.7038 & 0.9850 & 0.0326 \\
\hline AC073896.2 & 0.7519 & 0.5795 & 0.9755 & 0.0318 & 0.5455 & 0.3846 & 0.7736 & 0.0007 \\
\hline AC009506.1 & 0.7479 & 0.5960 & 0.9386 & 0.0122 & 0.6740 & 0.4885 & 0.9298 & 0.0163 \\
\hline SGMS1-AS1 & 0.7370 & 0.5463 & 0.9942 & 0.0457 & 0.7119 & 0.5489 & 0.9233 & 0.0104 \\
\hline AC138356.1 & 0.7351 & 0.5439 & 0.9936 & 0.0453 & 0.7396 & 0.5989 & 0.9135 & 0.0051 \\
\hline AC008966.1 & 0.7348 & 0.5746 & 0.9397 & 0.0140 & 0.7491 & 0.5969 & 0.9402 & 0.0127 \\
\hline LINC00242 & 0.7280 & 0.5712 & 0.9279 & 0.0103 & 0.6744 & 0.5436 & 0.8366 & 0.0003 \\
\hline ST7-AS1 & 0.7146 & 0.5571 & 0.9167 & 0.0082 & 0.7633 & 0.6110 & 0.9534 & 0.0173 \\
\hline WDFY3-AS2 & 0.7067 & 0.5573 & 0.8961 & 0.0042 & 0.7835 & 0.6359 & 0.9655 & 0.0221 \\
\hline ZNF710-AS1 & 0.6972 & 0.5354 & 0.9079 & 0.0074 & 0.7130 & 0.5704 & 0.8912 & 0.0030 \\
\hline PXN-AS1 & 0.6910 & 0.5026 & 0.9501 & 0.0229 & 0.6138 & 0.4550 & 0.8279 & 0.0014 \\
\hline AC036176.1 & 0.6817 & 0.5213 & 0.8915 & 0.0051 & 0.6414 & 0.5054 & 0.8140 & 0.0003 \\
\hline AC016876.1 & 0.6378 & 0.4634 & 0.8778 & 0.0058 & 0.6581 & 0.4901 & 0.8838 & 0.0054 \\
\hline
\end{tabular}

IncRNAs marked with bold font were risky IncRNAs and others were protective IncRNAs.

a $p$-value of less than 0.05 was deemed to be statistically significant.

\section{RESULTS}

\section{Identification of Ferroptosis-Related IncRNAs}

Through the "GENCODE" database, we pinpointed 15071 and 11861 lncRNAs in the TCGA and ICGC datasets separately. Then, we obtained the expression matrix of 38 ferroptosisrelated genes from each dataset. Pearson correlation analysis was first carried out to explore ferroptosis-related lncRNAs, and thenceforth the univariate Cox regression was constructed to seek ferroptosis-related prognostic lncRNA $(p<0.05)$ in each dataset. Finally, we identified twenty-three ferroptosisrelated IncRNAs, which dramatically correlated with the survival among both datasets. The association between 23 ferroptosis-related lncRNAs and 38 ferroptosis-related genes was displayed in Figure 2. The univariate Cox analysis of twenty-three ferroptosis-related lncRNAs was shown in Table 1.

\section{Construction of the Ferroptosis-Related IncRNA Prognostic Marker in the The Cancer Genome Atlas Dataset}

The LASSO-Cox analysis was performed using the expression matrix of the twenty-three ferroptosis-related prognostic lncRNA mentioned above. The Fe-LPM encompassed eight ferroptosis-related lncRNAs in the TCGA dataset was identified (Figures 3A,B), the coefficient of the Fe-LPM was shown in Figure 3C. Based on TCGA dataset, a risk score was computed mentioned in the Materials and Methods part, and patients were then split for survival analysis into high-risk and low-risk subgroups on the threshold of median risk scores. The principal component analysis (PCA) indicated that we obtain a high degree of discrimination between high-risk and low-risk subgroups in the TCGA cohort (Supplementary Figures S1A, B), showing a remarkable difference between these different subgroups. KM survival curves considered that patients from the high-risk subgroup had considerably worse clinical outcomes than those from the low-risk subgroup (Figure 3D). The distribution of the risk score was shown in Figure 3E. ROC curves appraised the forecasting capability of the Fe-LPM for overall survival, and the area under the curve (AUC) reached 0.70 at 1 year, 0.71 at 2 years, 0.75 at 3 years (Figure 3F).

\section{Validation of the Ferroptosis-Related IncRNA Prognostic Marker in the International Cancer Genome Consortium Dataset}

To verify the stability of the prognostic markers constructed in the TCGA dataset, patients in the ICGC validation set were also assigned into high-risk and low-risk subgroups relative to the median risk scores calculated with the same formula as that from the training set. PCA in ICGC datasets was shown in Supplementary Figures S1C, D. Similar to the conclusion in the TCGA cohort, patients with highrisk scores had shorter OS than the low-risk ones in the ICGC dataset (Figure 3G). The distribution of the risk score was shown in 

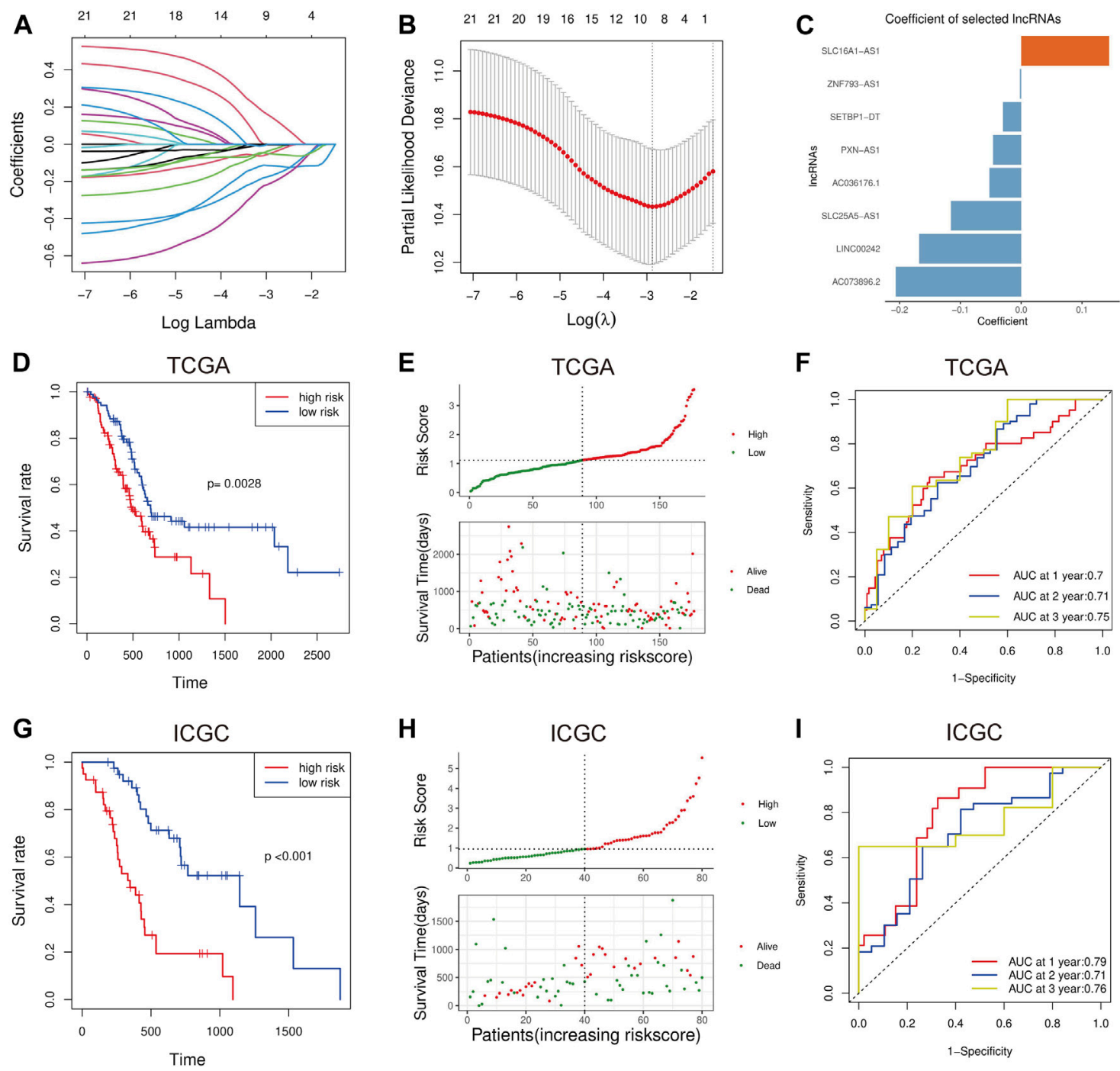

FIGURE 3 | The least absolute shrinkage and selection operator Cox (LASSO-Cox) analysis was implemented (A, B), and the coefficients were calculated by the 10-fold cross-validation based on minimum criteria (C). Kaplan-Meier analysis suggests the OS in the high-risk and low-risk groups in the TCGA cohort (D). Distribution of risk scores and survival status of PC patients in the TCGA cohort (E). Receiver operating characteristic (ROC) curve analysis for Fe-LPM in the TCGA cohort (F). In the ICGC validation cohort, the results of the Kaplan-Meier analysis were consistent with those in the TCGA cohort (G). Distribution of risk scores and survival status of PC patients in the ICGC cohort (H). Receiver operating characteristic (ROC) curve analysis for Fe-LPM in the ICGC validation cohort (I).

Figure 3H. The AUC of the Fe-LPM in the ICGC cohort was 0.79 at 1 year, 0.71 at 2 years, 0.76 at 3 years (Figure $3 \mathrm{I}$ ).

\section{Prognostic Analysis of the Eight Ferroptosis-Related IncRNA Prognostic Marker}

We implemented the univariate Cox analysis to estimate the prognostic role of the eight Fe-LPM. The result indicates that SLC16A1-AS1 is a risk factor with HR $>1$, whereas SETBP1DT, ZNF93-AS1, SLC25A5-AS1, AC073896.2, LINC00242, PXNAS1 and AC036176.1 are protective factors (Figure 4A). The heatmap exhibits that SLC16A1-AS1 expression increased with increasing risk score, while SETBP1-DT, ZNF93-AS1, SLC25A5-
AS1, AC073896.2, LINC00242, PXN-AS1 and AC036176.1 expression decreased with increasing risk score (Figure 4B). The Kaplan-Meier curve certified that lower expression of SLC16A1-AS1 and higher expression of SETBP1-DT, ZNF93-AS1, SLC25A5-AS1, AC073896.2, LINC00242, PXN-AS1 and AC036176.1 corresponded with higher survival time in the TCGA cohort (Figures 4C-J).

\section{Clinical Relevance of the Ferroptosis-Related IncRNA Prognostic Marker}

To clarify the associations between risk scores and clinicopathological characteristics, we further scrutinize the clinicopathological information from the TCGA cohort. The result manifested that 
A

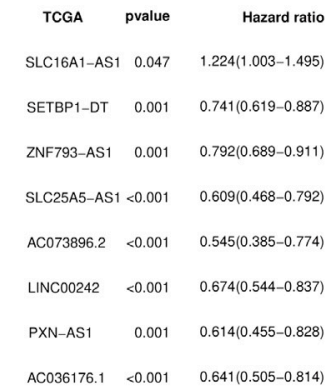

B

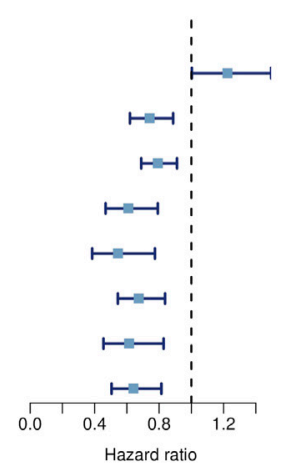

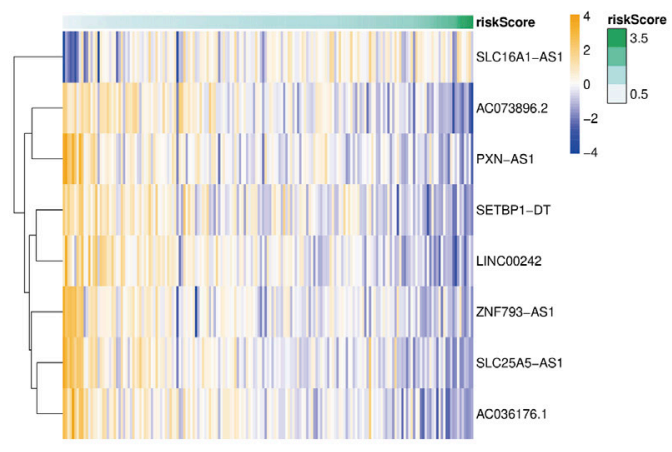

C

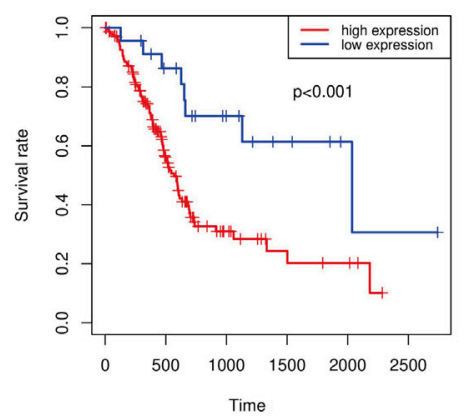

$\mathbf{F}$

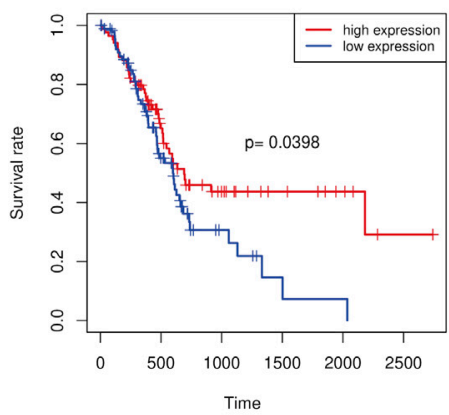

D

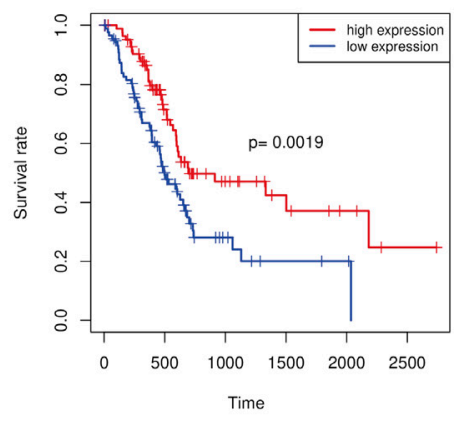

$\mathbf{G}$

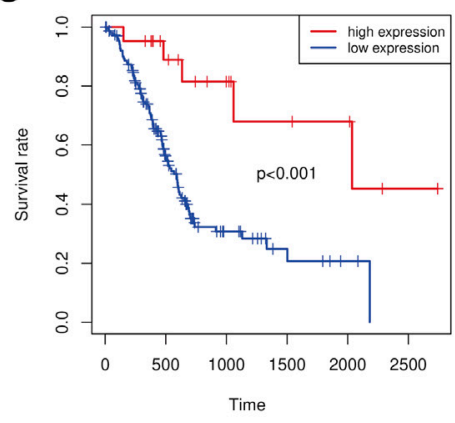

E
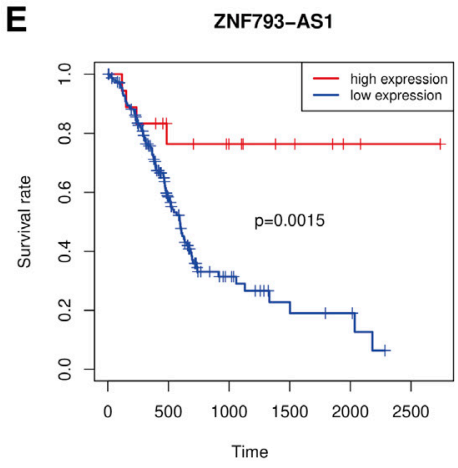

H

LINC00242

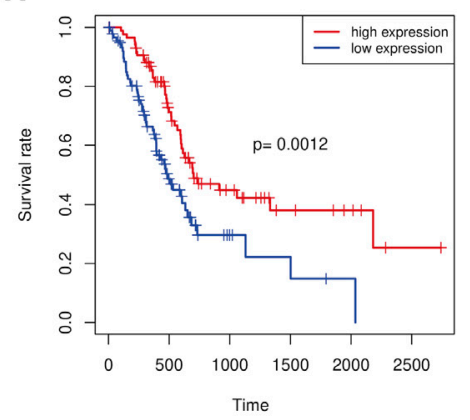

I

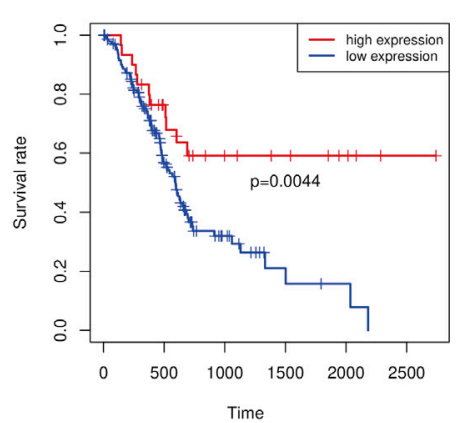

J $\quad$ AC036176.1

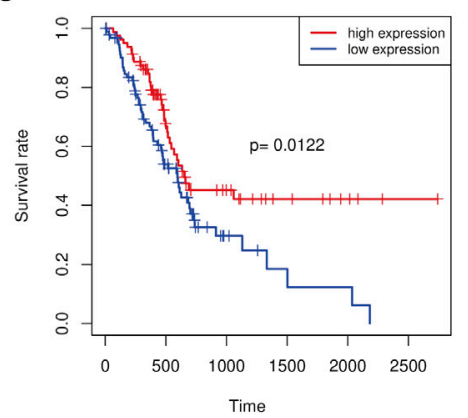

FIGURE 4 | Forest plot of the univariate Cox analysis in eight Fe-LPM (A). Heatmap showed the relationship between risk score and expression of the eight Fe-LPM (B). Kaplan-Meier analysis in different expression subgroups of the eight Fe-LPM (C-J). 

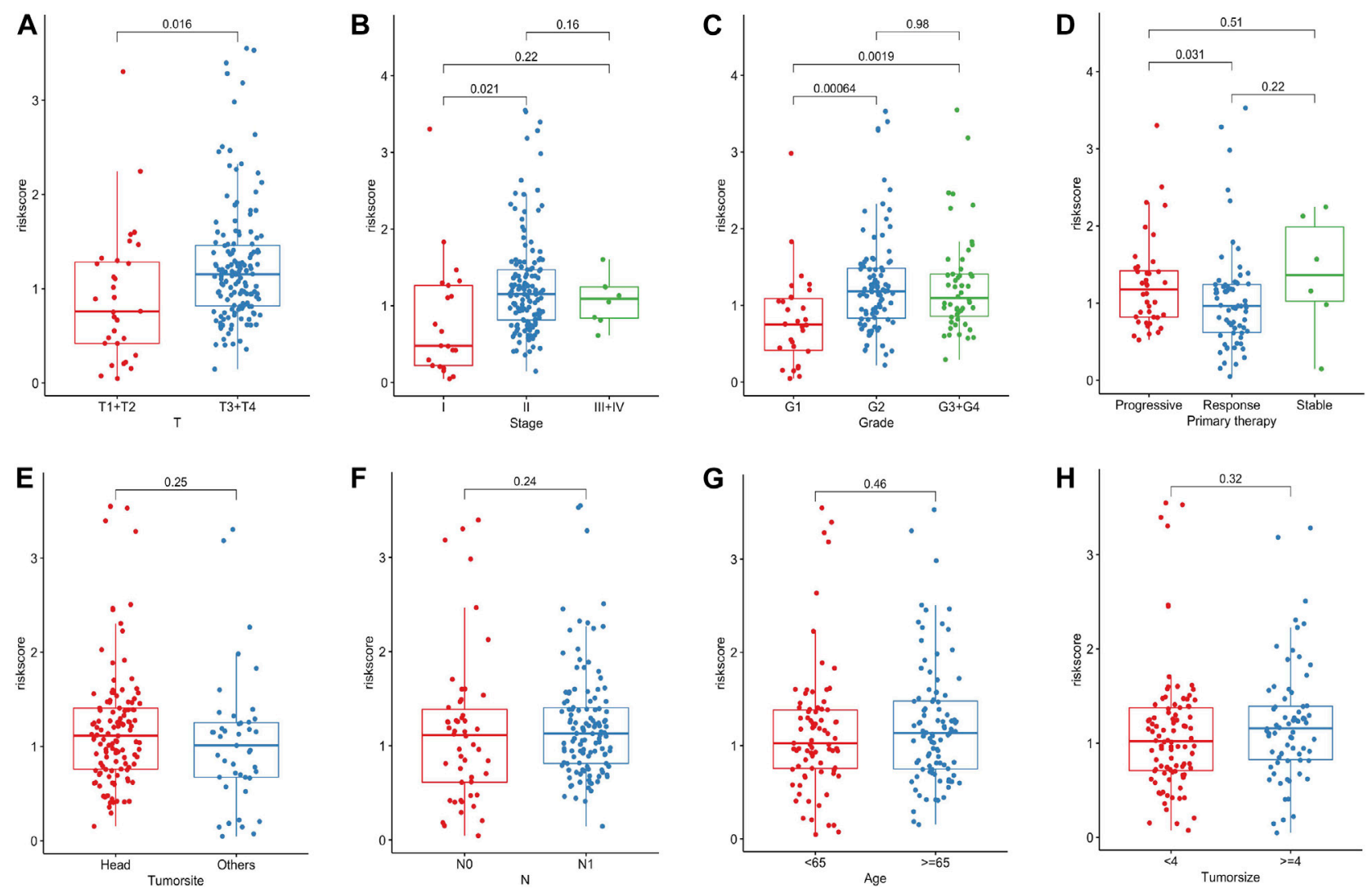

FIGURE 5 | The risk score significantly correlated with pathological T stage, TNM stage, grade and primary therapy outcome of PC patients (A-D). The risk score was not related to the tumor site, pathological $\mathrm{N}$ stage, age and tumor size $\mathbf{( E - H )}$.

the risk score apparently corresponded with pathological $\mathrm{T}$ stage, TNM stage, grade and primary therapy outcome of PC patients (Figures 5A-D). The stronger the tendency to higher pathological $\mathrm{T}$ stage, the higher the risk scores, which may explain the poorer survival rate of the high-risk group. Consistently, we found that risk scores were higher in advanced PC than those in early TNM stages and grades. Furthermore, patients with progressive status after primary therapy obtained higher risk scores than those with responsive status, suggesting that this Fe-LPM may serve as a potential evaluation index of primary therapy in PC patients. In contrast, the tumor site, pathological $\mathrm{N}$ stage, age and tumor size proved nonsensical (Figures 5E-H).

\section{Pathway Enrichment Analysis and Gene Set Enrichment Analysis Analysis in the The Cancer Genome Atlas Dataset}

For exploring the underlying pathway among the high-risk and low-risk subgroups, 1213 differentially expressed mRNAs (DEmRNAs) between high-risk and low-risk groups were selected according to the criteria of $\mid \log _{2}$ (Fold change) $\mid>1$ and $p<0.05$. These DEmRNAs were significantly enriched in chemical synaptic transmission, regulation of hormone levels, presynapse and regulation of ion transport (Supplementary Figure S2). The results of GSEA revealed that the genes in the low-risk subgroup were significantly enriched in fatty acid metabolism, peroxisome, oxidative phosphorylation, PI3KAKT-mTOR signaling and lysosome (Figures 6A-F).

\section{The Independence of the}

\section{Ferroptosis-Related IncRNA Prognostic Marker in Predicting Overall Survival in Pancreatic Cancer}

To evaluate whether the Fe-LPM was an independent prognostic indicator for OS, univariate and multivariate Cox regression analyses were implemented. In the TCGA dataset, both univariate Cox analysis and multivariate Cox analysis showed that Fe-LPM was markedly correlated with survival status (Figures 7A,B). To establish a predictive tool for quantitative analysis of OS in PC patients, we initiated a prognostic nomogram based on the pathological $\mathrm{T}$ stage, risk score, primary therapy outcome and age in the TCGA cohort (Figure 7C). Calibration plots showed that the predictive concordance of this nomogram (Figure 7D).

\section{Construction of the Competing Endogenous RNA Regulatory Network}

The ceRNA network comprising lncRNAs, miRNAs and mRNAs was constructed for the sake of systematically probing into the 
A

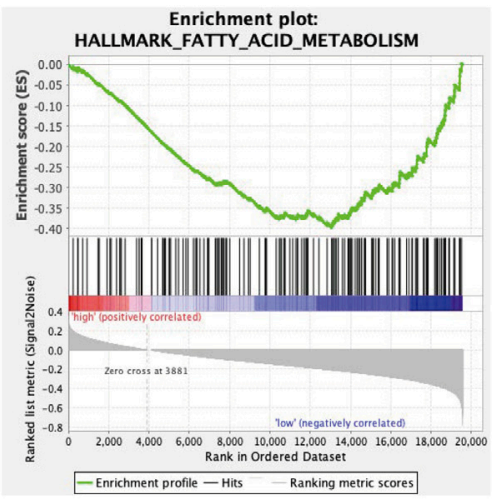

D

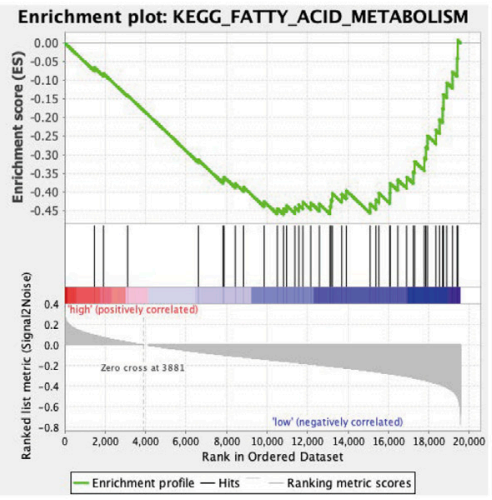

B

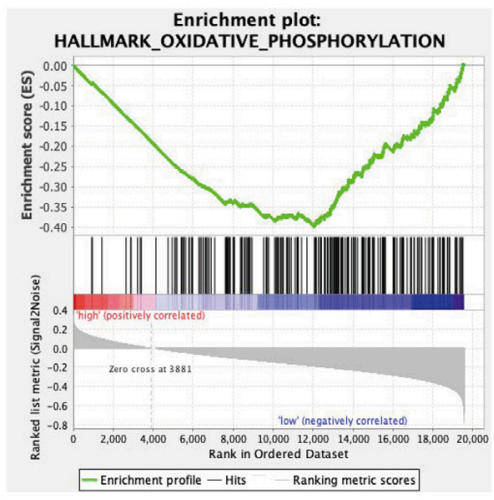

E

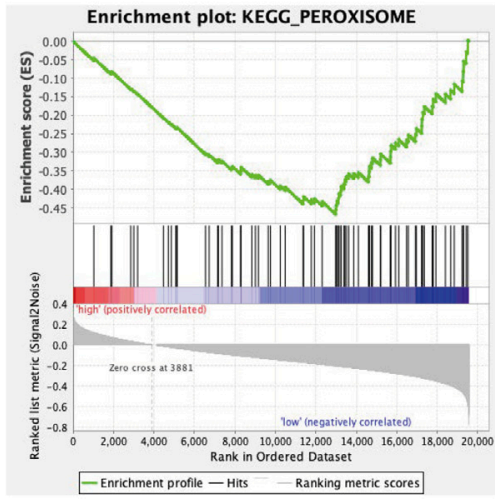

C

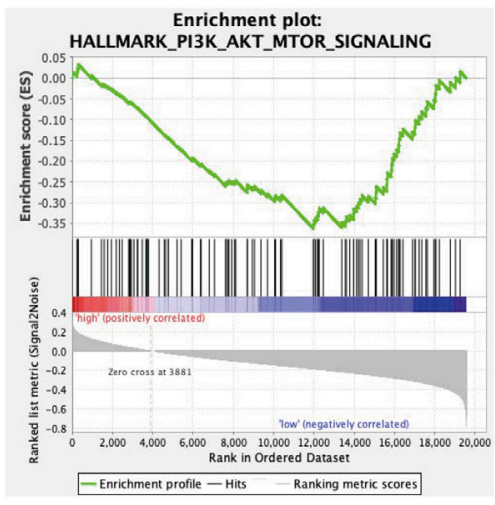

$\mathbf{F}$

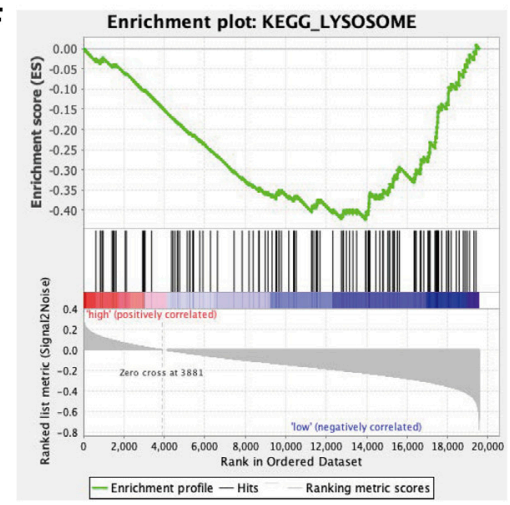

FIGURE 6 | GSEA illustrated that genes in the low-risk subgroup were significantly enriched in fatty acid metabolism (A, D), oxidative phosphorylation (B), PI3KAKT-mTOR signaling (C), peroxisome (E) and lysosome (F).

potential regulatory mechanism. According to those mentioned above twenty-three ferroptosis-related lncRNAs, four of twentythree lncRNAs were filtered from the miRcode database, and twenty-seven target miRNAs were distinguished. Then we use the MiRDB, miRTarBase, StarBase and TargetScan databases to extracted fifty-seven target mRNAs on account of twentyseven miRNAs. At length, four lncRNAs, twenty-seven miRNAs and fifty-seven mRNAs were included in the ceRNA network (Figure 8A, Supplementary Figure S3). The pathway enrichment analysis of fifty-seven target mRNAs elucidated that these mRNAs were enriched in lipid transport, anion transport and dephosphorylation (Figures 8B-D), suggesting that the FeLPM was definitely correlated with the process of ferroptosis.

\section{DISCUSSION}

Ferroptosis, an extraordinary cell death pathway driven by irondependent lipid peroxidation, regulates the epigenetic program and metabolic changes in numerous cancers (Wu et al., 2020). Multiple studies have already shown that specific lncRNAs can alleviate the malignancy of tumors by regulating the ferroptosis-related pathway. Interacted with G3BP1, the lncRNA P53RRA could restrain lung cancer progression via activating the p53 signaling pathway which correlated with ferroptosis (Mao et al., 2018). LncRNA GABPB1AS1 inhibits the translation of GABPB1, causing the accumulation of iron-dependent reactive oxygen species (ROS) and improving the overall survival of HCC patients (Qi et al., 2019). Additionally, most studies have confirmed that lncRNAs might function as ceRNA of mRNAs, thereby manipulating ferroptosis-mediated tumor progression. LINC00336 functions as an oncogene in lung cancer and regulates cystathionine- $\beta$-synthase (CBS) expression by competing for miR6852 (Wang et al., 2019). The lncRNA MT1DP could sensitize NSCLC cells to erastin-induced ferroptosis by regulating the miR-365a-3p/NRF2 axis, mitigating the mortality of NSCLC (Gai et al., 2020). After pondering these clues, we hypothesize that ferroptosis is tightly linked to lncRNAs, and we ought to concentrate on the potential interaction between lncRNAs and ferroptosis to uncover underlying prognostic biomarkers.

In the aggregate, $257 \mathrm{PC}$ patients were involved in detecting the prognostic value of ferroptosis-related lncRNAs. Twentythree ferroptosis-related prognostic lncRNAs were identified, and eight of them were singled out as the Fe-LPM. LNC00242 was high-expressed in gastric cancer (GC) tissue regulated by LINC00242/miR-1-3p/G6PD axis (Deng et al., 2021), and it may be a promising biomarker of GC (Zhong et al., 2020). Mediated by E2F1, SLC16A1-AS1 could progress bladder cancer to the invasive stage (Logotheti et al., 2020) and serve as a new diagnostic indicator in hepatocellular carcinoma (Song et al., 2019; Pei et al., 2020). It has been reported that SLC25A5-AS1 functions as a suppressor in the progression of gastric cancer to 

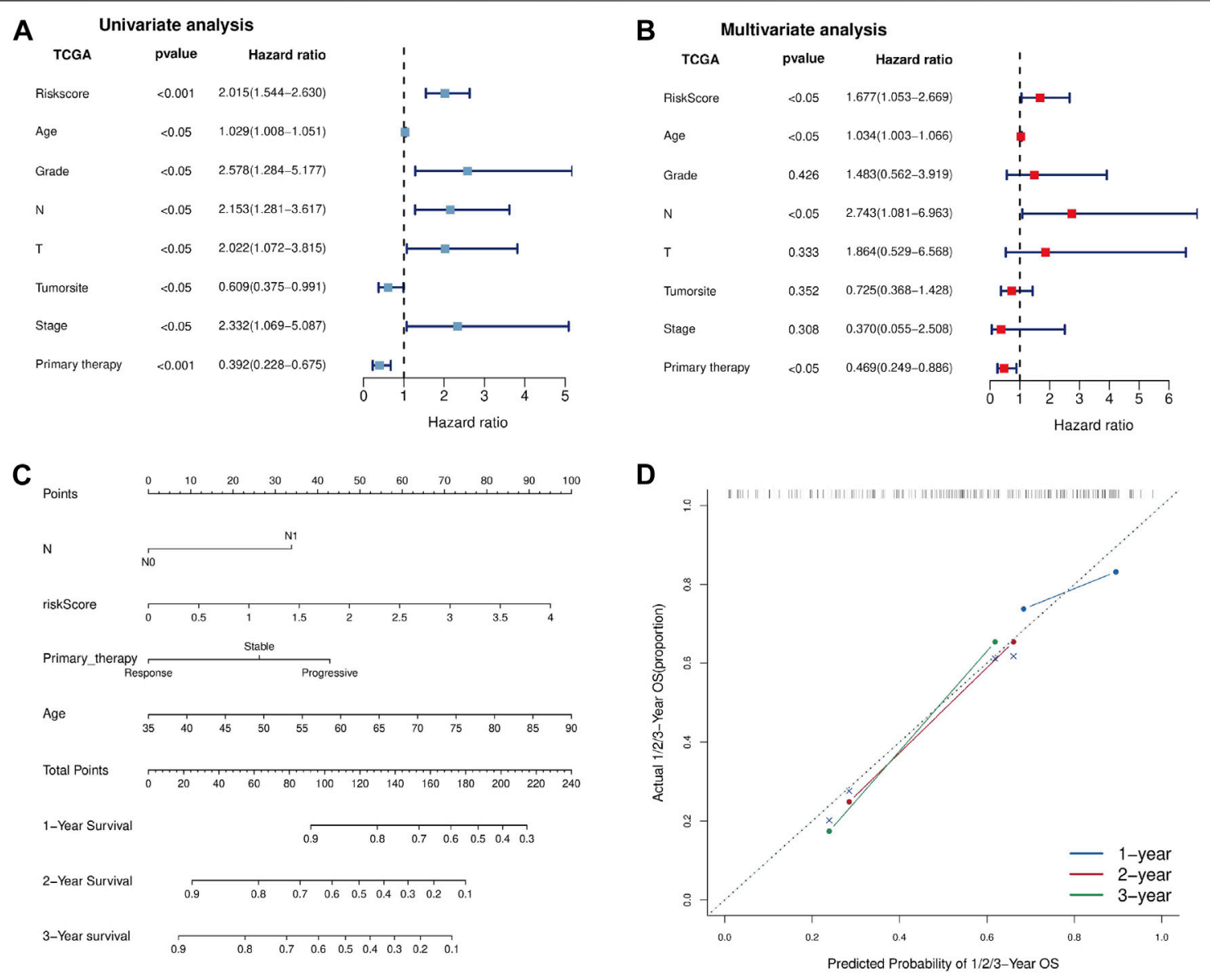

FIGURE 7 |Univariate and multivariate Cox analyses were performed based on risk scores and other clinical features in the TCGA dataset (A, B). A nomogram was constructed based on pathological T stage, risk score, primary therapy outcome and age in the TCGA cohort (C). A calibration plot confirmed the robustness of the FeLPM (D).

regulate cellular behaviors via the miR-19a-3p/PTEN/PI3K/AKT signaling pathway (Li et al., 2019). PXN-AS1 stifles PC progression by serving as a sponge to suppress the expression of miR-3064 (Yan et al., 2019) and it also plays vital roles in hepatocarcinogenesis as a prognostic biomarker (Yuan et al., 2017). Since half of the eight lncRNAs were reported to be associated with tumor progression, the others need to be further determined their mechanism in the process of ferroptosis by experiments in our future studies.

According to the median risk score calculated by the coefficient of eight Fe-LPM, the PC patients were stratified into the high-risk and low-risk subgroups. To further investigate the biological process and pathway between the two subgroups, GSEA revealed fatty acid metabolism, peroxisome, oxidative phosphorylation, PI3K-AKT-mTOR signaling and lysosome were enormously enriched in the lowrisk subgroup. These signaling pathways have been confirmed to be indispensable for ferroptosis (Stockwell et al., 2017), implying that the low-risk subgroup is more sensitive to ferroptosis. Polyunsaturated fatty acid (PUFA) biosynthesis pathway has been reported to be a marker for predicting the ability of ferroptosis-mediated therapy in gastric cancer (Lee et al., 2020). Peroxisome and oxidative phosphorylation also participate in the biological process of ferroptosis (Tang and Kroemer, 2020; Yusuf et al., 2020). Mutation of PI3K-AKTmTOR signaling protects cancer cells from ferroptosis death through SREBP1/SCD1-mediated lipogenesis (Yi et al., 2020). Besides, various studies have reported that ferroptosis can mitigate gemcitabine resistance and lead to a favorable prognosis in pancreatic cancer cells (Ye et al., 2020; Ye et al., 2021), which is consistent with the result of our study: most of the Fe-LPM are protective factors based on the univariate Cox regression analysis.

Predicting clinical outcome endpoints is necessary for personalized and translational medicine (Behrens et al., 2017). Although numerous efforts have been exerted to research novel drugs that can suppress the PC, we can only expect to successfully translate genomic findings into clinical practice through the development of genome-sequencing technology (Singh et al., 2019). Consequently, we made further efforts to 


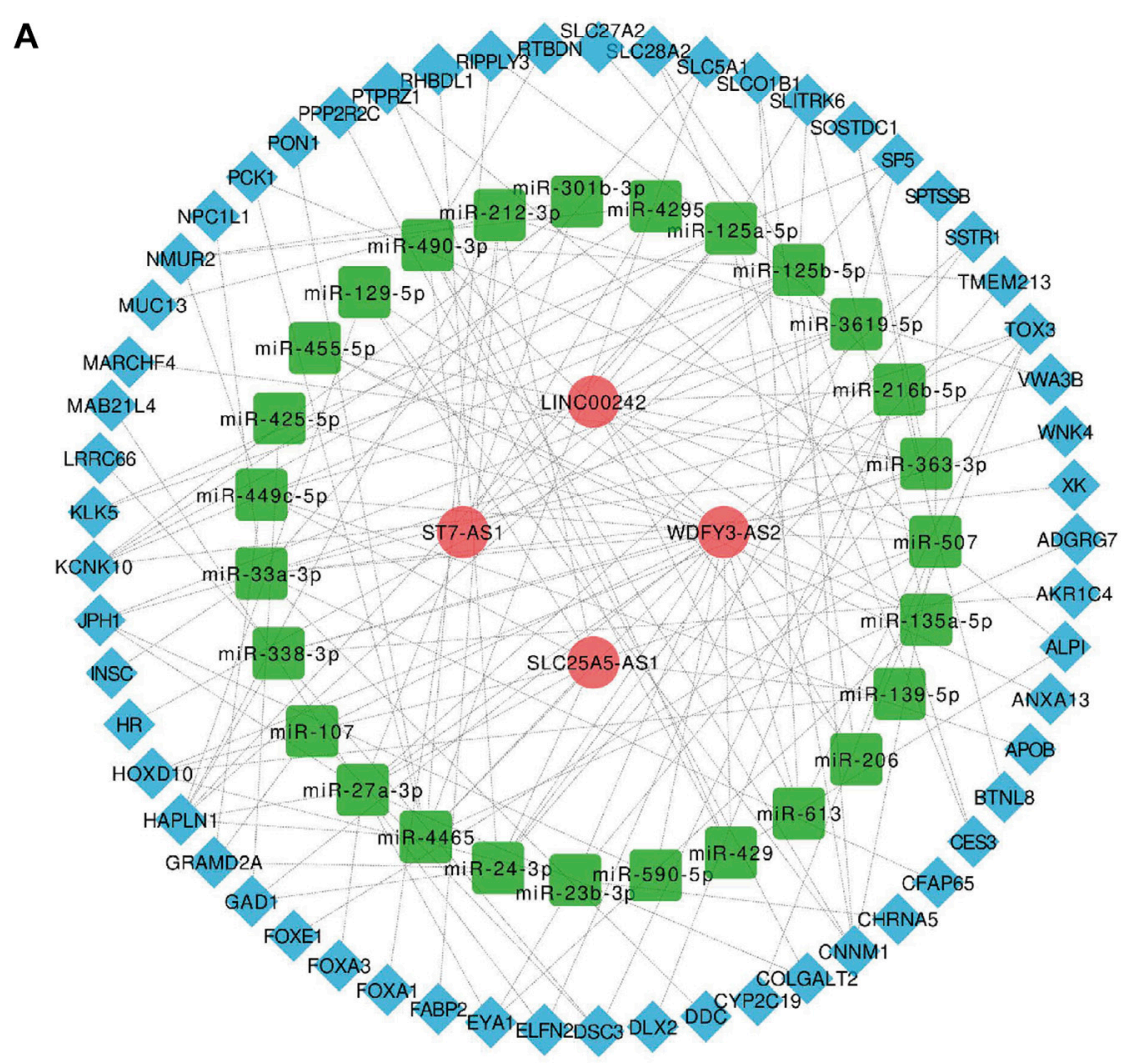

B

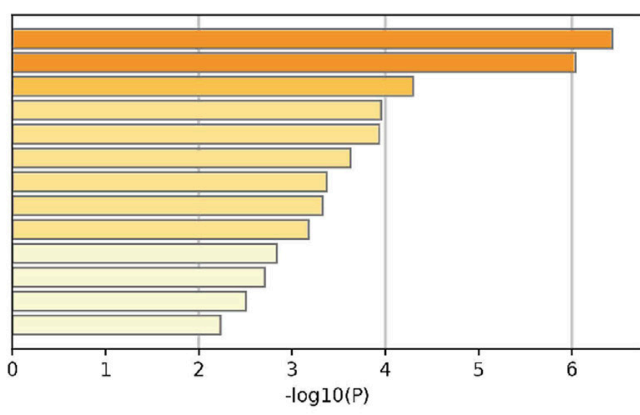

GO:0007586: digestion GO:0006869: lipid transport GO:0006820: anion transport GO:0007610: behavior

R-HSA-556833: Metabolism of lipids

GO:0048663: neuron fate commitment

k003320: PPAR signaling pathway

GO:0016311: dephosphorylation

GO:0042475: odontogenesis of dentin-containing tooth

GO:0006821: chloride transport

GO:0006836: neurotransmitter transport

GO:0031667: response to nutrient levels

GO:0071466: cellular response to xenobiotic stimulus
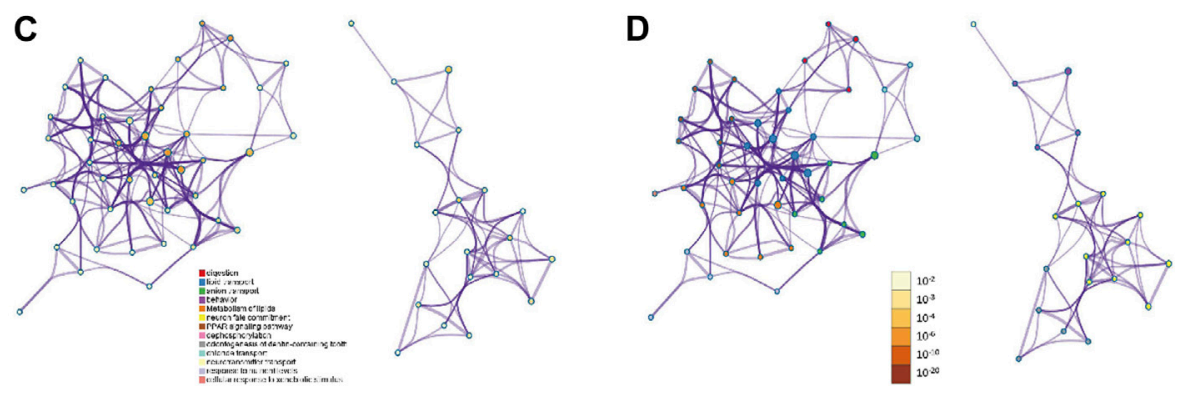

FIGURE 8 | A ceRNA network including 4 IncRNAs-27 miRNAs-57 mRNAs was established (A). The heatmap (B), cluster ID network (C) and p-value network (D) of enriched pathways based on 57 target mRNAs. 
explore the prognostic value of the Fe-LPM. The univariate and multivariate Cox analyses identified that Fe-LPM was an independent indicator for OS. A nomogram was based on four clinicopathological features (pathological T stage, TNM stage, grade and primary therapy outcome of PC patients) was constructed and validated by calibration plot. Afterward, a ceRNA network was established including four lncRNAs, twenty-seven miRNAs and fifty-seven mRNAs. The above results confirmed that Fe-LPM may propose new prospects and intervention measures for the treatment of $\mathrm{PC}$ and provides a robust theoretical basis for future clinical judgments.

Given all the results offered above, we concluded that the eight Fe-LPM (including SLC16A1-AS1, SETBP1-DT, ZNF93-AS1, SLC25A5-AS1, AC073896.2, LINC00242, PXN-AS1 and AC036176) prove to be a prognostic biomarker of OS for PC patients. Function prediction further confirmed the importance of Fe-LPM in PC, offering us a comparatively systematic and comprehensive knowledge of the links among ferroptosis, lncRNAs and PC. However, it is observed that the shortage of clinical information in ICGC_AU_PAAD leads to a lack of profound validations in the ICGC cohort. On the other hand, our study should make further validations in vivo and vitro soon.

\section{DATA AVAILABILITY STATEMENT}

The datasets presented in this study can be found in online repositories. The names of the repository/repositories and accession number(s) can be found in the article/ Supplementary Material.

\section{REFERENCES}

Behrens, D., Walther, W., and Fichtner, I. (2017). Pancreatic Cancer Models for Translational Research. Pharmacol. Ther. 173, 146-158. doi:10.1016/j. pharmthera.2017.02.013

Dai, E., Han, L., Liu, J., Xie, Y., Zeh, H. J., Kang, R., et al. (2020). Ferroptotic Damage Promotes Pancreatic Tumorigenesis through a TMEM173/STINGdependent DNA Sensor Pathway. Nat. Commun. 11 (1), 6339. doi:10.1038/ s41467-020-20154-8

Deng, P., Li, K., Gu, F., Zhang, T., Zhao, W., Sun, M., et al. (2021). LINC00242/ miR-1-3p/G6PD axis Regulates Warburg Effect and Affects Gastric Cancer Proliferation and Apoptosis. Mol. Med. 27 (1), 9. doi:10.1186/s10020-02000259-y

Dreyer, S. B., Chang, D. K., Bailey, P., and Biankin, A. V. (2017). Pancreatic Cancer Genomes: Implications for Clinical Management and Therapeutic Development. Clin. Cancer Res. 23 (7), 1638-1646. doi:10.1158/1078-0432. Ccr-16-2411

Gai, C., Liu, C., Wu, X., Yu, M., Zheng, J., Zhang, W., et al. (2020). MT1DP Loaded by Folate-Modified Liposomes Sensitizes Erastin-Induced Ferroptosis via Regulating miR-365a-3p/NRF2 axis in Non-small Cell Lung Cancer Cells. Cell Death Dis 11 (9), 751. doi:10.1038/s41419-020-02939-3

Hassannia, B., Vandenabeele, P., and Vanden Berghe, T. (2019). Targeting Ferroptosis to Iron Out Cancer. Cancer Cell 35 (6), 830-849. doi:10.1016/j. ccell.2019.04.002

Lee, J.-Y., Nam, M., Son, H. Y., Hyun, K., Jang, S. Y., Kim, J. W., et al. (2020). Polyunsaturated Fatty Acid Biosynthesis Pathway Determines Ferroptosis Sensitivity in Gastric Cancer. Proc. Natl. Acad. Sci. USA 117 (51), 32433-32442. doi:10.1073/pnas.2006828117

Li, N., Yang, G., Luo, L., Ling, L., Wang, X., Shi, L., et al. (2020). lncRNA THAP9AS1 Promotes Pancreatic Ductal Adenocarcinoma Growth and Leads to a Poor

\section{AUTHOR CONTRIBUTIONS}

Conceptualization, XY and HZ; Methodology, DC and WG; Software, DC; Supervision, WG, LZ and HZ; Validation, WG, $\mathrm{XY}$ and HZ; Writing-original draft, DC; Writing-review and editing, WG, LZ, XZ and ZL.

\section{FUNDING}

This research was funded by the National Natural Science Foundation of China (NO.81873589); National Natural Science Foundation For Young Scientists of China (NO.82000614); National Natural Science Foundation for Young Scientists of Hunan Province, China (2020JJ5876); Science and Technology Project of Changsha, Hunan, China (NO.kq2004146).

\section{ACKNOWLEDGMENTS}

We would like to exert compelling appreciation for the TCGA and ICGC projects.

\section{SUPPLEMENTARY MATERIAL}

The Supplementary Material for this article can be found online at: https://www.frontiersin.org/articles/10.3389/fcell.2022.819724/ full\#supplementary-material

Clinical Outcome via Sponging miR-484 and Interacting with YAP. Clin. Cancer Res. 26 (7), 1736-1748. doi:10.1158/1078-0432.Ccr-19-0674

Li, X., Yan, X., Wang, F., Yang, Q., Luo, X., Kong, J., et al. (2019). Down-regulated lncRNA SLC25A5-AS1 Facilitates Cell Growth and Inhibits Apoptosis via miR19a-3p/PTEN/PI3K/AKT Signalling Pathway in Gastric Cancer. J. Cel Mol Med 23 (4), 2920-2932. doi:10.1111/jcmm.14200

Logotheti, S., Marquardt, S., Gupta, S. K., Richter, C., Edelhäuser, B. A. H., Engelmann, D., et al. (2020). LncRNA-SLC16A1-AS1 Induces Metabolic Reprogramming during Bladder Cancer Progression as Target and Coactivator of E2F1. Theranostics 10 (21), 9620-9643. doi:10.7150/thno. 44176

Mao, C., Wang, X., Liu, Y., Wang, M., Yan, B., Jiang, Y., et al. (2018). A G BP1Interacting lncRNA Promotes Ferroptosis and Apoptosis in Cancer via Nuclear Sequestration of P53. Cancer Res. 78 (13), 3484-3496. doi:10.1158/0008-5472. Can-17-3454

Neoptolemos, J. P., Kleeff, J., Michl, P., Costello, E., Greenhalf, W., and Palmer, D. H. (2018). Therapeutic Developments in Pancreatic Cancer: Current and Future Perspectives. Nat. Rev. Gastroenterol. Hepatol. 15 (6), 333-348. doi:10.1038/s41575-018-0005-x

Pei, S., Chen, Z., Tan, H., Fan, L., Zhang, B., and Zhao, C. (2020). SLC16A1-AS1 Enhances Radiosensitivity and Represses Cell Proliferation and Invasion by Regulating the miR-301b-3p/CHD5 axis in Hepatocellular Carcinoma. Environ. Sci. Pollut. Res. 27 (34), 42778-42790. doi:10.1007/s11356-020-09998-1

Qi, W., Li, Z., Xia, L., Dai, J., Zhang, Q., Wu, C., et al. (2019). LncRNA GABPB1AS1 and GABPB1 Regulate Oxidative Stress during Erastin-Induced Ferroptosis in HepG2 Hepatocellular Carcinoma Cells. Sci. Rep. 9 (1), 16185. doi:10.1038/s41598-019-52837-8

Ren, X., Chen, C., Luo, Y., Liu, M., Li, Y., Zheng, S., et al. (2020). IncRNA-PLACT1 Sustains Activation of NF-Kb Pathway through a Positive Feedback Loop with IкBa/E2F1 axis in Pancreatic Cancer. Mol. Cancer 19 (1), 35. doi:10.1186/ s12943-020-01153-1 
Singh, R. R., Goldberg, J., Varghese, A. M., Yu, K. H., Park, W., and O’Reilly, E. M. (2019). Genomic Profiling in Pancreatic Ductal Adenocarcinoma and a Pathway towards Therapy Individualization: A Scoping Review. Cancer Treat. Rev. 75, 27-38. doi:10.1016/j.ctrv.2019.03.003

Song, M., Zhong, A., Yang, J., He, J., Cheng, S., Zeng, J., et al. (2019). Large-scale Analyses Identify a Cluster of Novel Long Noncoding RNAs as Potential Competitive Endogenous RNAs in Progression of Hepatocellular Carcinoma. Aging 11 (22), 10422-10453. doi:10.18632/aging.102468

Stockwell, B. R., Friedmann Angeli, J. P., Bayir, H., Bush, A. I., Conrad, M., Dixon, S. J., et al. (2017). Ferroptosis: A Regulated Cell Death Nexus Linking Metabolism, Redox Biology, and Disease. Cell 171 (2), 273-285. doi:10.1016/ j.cell.2017.09.021

Stockwell, B. R., Jiang, X., and Gu, W. (2020). Emerging Mechanisms and Disease Relevance of Ferroptosis. Trends Cel Biol. 30 (6), 478-490. doi:10.1016/j.tcb. 2020.02.009

Sun, Y., and Ma, L. (2019). New Insights into Long Non-coding RNA MALAT1 in Cancer and Metastasis. Cancers 11 (2), 216. doi:10.3390/cancers11020216

Tang, D., Chen, X., Kang, R., and Kroemer, G. (2021). Ferroptosis: Molecular Mechanisms and Health Implications. Cell Res 31 (2), 107-125. doi:10.1038/ s41422-020-00441-1

Tang, D., and Kroemer, G. (2020). Peroxisome: the New Player in Ferroptosis. Sig Transduct Target. Ther. 5 (1), 273. doi:10.1038/s41392-020-00404-3

Vincent, A., Herman, J., Schulick, R., Hruban, R. H., and Goggins, M. (2011). Pancreatic Cancer. The Lancet 378 (9791), 607-620. doi:10.1016/s01406736(10)62307-0

Wang, M., Mao, C., Ouyang, L., Liu, Y., Lai, W., Liu, N., et al. (2019). Long Noncoding RNA LINC00336 Inhibits Ferroptosis in Lung Cancer by Functioning as a Competing Endogenous RNA. Cell Death Differ 26 (11), 2329-2343. doi:10.1038/s41418-019-0304-y

Wolfgang, C. L., Herman, J. M., Laheru, D. A., Klein, A. P., Erdek, M. A., Fishman, E. K., et al. (2013). Recent Progress in Pancreatic Cancer. CA A Cancer J. Clinicians 63 (5), 318-348. doi:10.3322/caac.21190

Wu, Y., Zhang, S., Gong, X., Tam, S., Xiao, D., Liu, S., et al. (2020). The Epigenetic Regulators and Metabolic Changes in Ferroptosis-Associated Cancer Progression. Mol. Cancer 19 (1), 39. doi:10.1186/s12943-020-01157-x

Xiong, G., Feng, M., Yang, G., Zheng, S., Song, X., Cao, Z., et al. (2017). The Underlying Mechanisms of Non-coding RNAs in the Chemoresistance of Pancreatic Cancer. Cancer Lett. 397, 94-102. doi:10.1016/j.canlet.2017. 02.020

Xiong, G., Liu, C., Yang, G., Feng, M., Xu, J., Zhao, F., et al. (2019). Long Noncoding RNA GSTM3TV2 Upregulates LAT2 and OLR1 by Competitively Sponging Let-7 to Promote Gemcitabine Resistance in Pancreatic Cancer. J. Hematol. Oncol. 12 (1), 97. doi:10.1186/s13045-019-0777-7

Yan, J., Jia, Y., Chen, H., Chen, W., and Zhou, X. (2019). Long Non-coding RNA PXN-AS1 Suppresses Pancreatic Cancer Progression by Acting as a Competing Endogenous RNA of miR-3064 to Upregulate PIP4K2B Expression. J. Exp. Clin. Cancer Res. 38 (1), 390. doi:10.1186/s13046-019-1379-5
Ye, Z., Hu, Q., Zhuo, Q., Zhu, Y., Fan, G., Liu, M., et al. (2020). Abrogation of ARF6 Promotes RSL3-Induced Ferroptosis and Mitigates Gemcitabine Resistance in Pancreatic Cancer Cells. Am. J. Cancer Res. 10 (4), 1182-1193.

Ye, Z., Zhuo, Q., Hu, Q., Xu, X., Mengqi liu, L., Zhang, Z., et al. (2021). FBW7NRA41-SCD1 axis Synchronously Regulates Apoptosis and Ferroptosis in Pancreatic Cancer Cells. Redox Biol. 38, 101807. doi:10.1016/j.redox.2020.101807

Yee, P. P., Wei, Y., Kim, S.-Y., Lu, T., Chih, S. Y., Lawson, C., et al. (2020). Neutrophil-induced Ferroptosis Promotes Tumor Necrosis in Glioblastoma Progression. Nat. Commun. 11 (1), 5424. doi:10.1038/s41467-020-19193-y

Yi, J., Zhu, J., Wu, J., Thompson, C. B., and Jiang, X. (2020). Oncogenic Activation of PI3K-AKT-mTOR Signaling Suppresses Ferroptosis via SREBP-Mediated Lipogenesis. Proc. Natl. Acad. Sci. USA 117 (49), 31189-31197. doi:10.1073/ pnas.2017152117

Yuan, J.-h., Liu, X.-n., Wang, T.-t., Pan, W., Tao, Q.-f., Zhou, W.-p., et al. (2017). The MBNL3 Splicing Factor Promotes Hepatocellular Carcinoma by Increasing PXN Expression through the Alternative Splicing of lncRNA-PXN-AS1. Nat. Cel Biol 19 (7), 820-832. doi:10.1038/ncb3538

Yusuf, R. Z., Saez, B., Sharda, A., van Gastel, N., Yu, V. W. C., Baryawno, N., et al. (2020). Aldehyde Dehydrogenase 3a2 Protects AML Cells from Oxidative Death and the Synthetic Lethality of Ferroptosis Inducers. Blood 136 (11), 1303-1316. doi:10.1182/blood.2019001808

Zhang, H., Deng, T., Liu, R., Ning, T., Yang, H., Liu, D., et al. (2020). CAF Secreted miR-522 Suppresses Ferroptosis and Promotes Acquired Chemo-Resistance in Gastric Cancer. Mol. Cancer 19 (1), 43. doi:10.1186/s12943-020-01168-8

Zheng, J., and Conrad, M. (2020). The Metabolic Underpinnings of Ferroptosis. Cel Metab. 32 (6), 920-937. doi:10.1016/j.cmet.2020.10.011

Zhong, X., Yu, X., Wen, X., Chen, L., and Gu, N. (2020). Activation of the LINC00242/miR-141/FOXC1 axis Underpins the Development of Gastric Cancer. Cancer Cel Int 20, 272. doi:10.1186/s12935-020-01369-7

Conflict of Interest: The authors declare that the research was conducted in the absence of any commercial or financial relationships that could be construed as a potential conflict of interest.

Publisher's Note: All claims expressed in this article are solely those of the authors and do not necessarily represent those of their affiliated organizations, or those of the publisher, the editors and the reviewers. Any product that may be evaluated in this article, or claim that may be made by its manufacturer, is not guaranteed or endorsed by the publisher.

Copyright (C) 2022 Chen, Gao, Zang, Zhang, Li, Zhu and Yu. This is an open-access article distributed under the terms of the Creative Commons Attribution License (CC $B Y)$. The use, distribution or reproduction in other forums is permitted, provided the original author(s) and the copyright owner(s) are credited and that the original publication in this journal is cited, in accordance with accepted academic practice. No use, distribution or reproduction is permitted which does not comply with these terms. 